Published in final edited form as:

Future Virol. 2010 September 1; 5(5): 539-543. doi:10.2217/fvl.10.43.

\title{
Retroviruses: Molecular Biology, Genomics and Pathogenesis
}

\author{
Alice Telesnitsky \\ Department of Microbiology \& Immunology, University of Michigan, MI 48109-5620, USA
}

\begin{abstract}
Retroviruses have been of enduring interest to biomedical scientists from the time Peyton Rous first reported that a filterable agent could transmit cancer in chickens, through to the current challenges and discoveries associated with HIV-1. This book represents an ambitious effort, spearheaded by editors Reinhard Kurth and Norbert Bannert, to produce a definitive reference for the field. Many review articles on individual topics covered are currently available elsewhere, as are several strong compendiums. However, no authoritative, current, comprehensive text covering the complex subject of retrovirology exists. Although some sections of old reference books hold up remarkably well, whole new areas of inquiry, such as host antiretro viral restriction factors, have blossomed in recent years, and rapid progress in fields, such as virus assembly, have made it clear that a volume similar to what the editors sought to deliver here is long overdue.
\end{abstract}

This book is comprised of 16 chapters, each authored by experts in their respective fields, which are listed and briefly reviewed later in this article. This book opens with a very brief preface, illustrated with a single dendrogram that presents the seven genera of retroviruses, followed by an extensive 'Abbreviations' key. Then, rather than start the volume with introductory information about replication cycles and general properties of retroviruses (which, ironically, is basically what the final chapter contains), the book starts with a chapter on nonretroviral retrotransposons.

\section{Everlasting war dance between retrotransposons \& their metazoan hosts: David Symer \& Jef Boeke}

This opening chapter describes retrotransposons, with its whimsical title alluding to recent research demonstrating that the genomes of metazoans not only contain mind-boggling numbers of retrotransposons (perhaps 1 million short interspersed elements per human haploid genome) but also a significant number of genes whose primary functions are to interfere with retrovirus and/or retrotransposon replication. The authors focused on recent developments and as such the chapter was less strong as an overview. Although an interesting read, the chapter fell prey to 'cuteness' in its descriptions of the genetic conflict between transposons and their hosts, and to entropy in its exuberant desire to toss in everything. The authors are clearly well versed in their field, but the chapter was repetitious and somewhat vague at points. Use of specialized terms, such as "exaptation", long before their definition made one wish this were an electronic version, so that the definition could be found. Little inaccuracies, such as the claim that each long terminal repeat contains a primer

(C) 2010 Future Medicine Ltd

Tel.: +1 734936 6466, Fax: +1 734764 3562, ateles@umich.edu.

Financial \& competing interests disclosure The author has no other relevant affiliations or financial involvement with any organization or entity with a financial interest in or financial conflict with the subject matter or materials discussed in the manuscript apart from those disclosed.

No writing assistance was utilized in the production of this manuscript. 
binding site, and the fact that this chapter is arguably the least retroviral of any here, made this a questionable choice for an introductory chapter.

\section{Endogenous retroviruses: Joachim Denner}

As Denner highlights, this century's genomics research has revealed that more human genetic space is devoted to human endogenous retroviruses than to human protein coding sequences. This chapter presents some of the neutral, beneficial and deleterious outcomes of endogenous retroviral insertions. Denner develops themes of host countermeasures to viruses and the use of virus functions by hosts, which he terms "enslavement". For example, syncytin, a human endogenous retrovirus envelope protein, provides fusion functions essential to human placental development. Other recent findings described here include how the notion that only "simple" retroviruses exist as endogenous retroelements was overturned by the discovery of lentivirus-like rabbit endogenous lentivirus type K (RELIK) elements in rabbits. Potential dangers of porcine endogenous retrovirus during xenotransplantation and possible endogenous retrovirus oncogene roles were also discussed. However, these pearls are minor portions of a long chapter rife with poor English and a somewhat confusing style. Denner proclaimed his focus to be recent publications, plus published and unpublished work from his own laboratory. As a result, although the excitement of the field was there, the field's foundations were not laid out clearly, a summary of the take home points was missing, and there were frequent detours into fairly raw data, such as of how many transcripts of unclear significance were upregulated in an unpublished microarray experiment, which made this lengthy chapter ramble.

\section{Retroviral particles, proteins \& genomes: Norbert Bannert, Uwe Fiebig \& Oliver Hohn}

This well-written chapter integrated information on the seven genera of retroviruses, both in overview and with specifics, with a focus on topics not covered elsewhere in the volume. This included a nice overview of major methods in the field, virus morphology and genome organization. The scope was encyclopedic without being overwhelming. In some places, such as when discussing the role of the central DNA flap of HIV, this flow was achieved by stating one side of a controversy without considering other viewpoints. The conclusions section did not summarize the chapter but, rather, presented prose that provided a bridge to the later chapters.

\section{Retroviral entry \& uncoating: Walther Mothes \& Pradeep Uchil}

These authors sought to provide the reader with a mechanistic understanding of the process of retroviral entry and uncoating. They presented the classes of fusion machinery used by different viruses and introduced type I fusion mechanics using the well-studied influenza hemagglutinin as a prototype. They then explained how most retroviruses achieve mechanistically similar outcomes with different cellular triggers, either in the absence of a $\mathrm{pH}$ drop or via the combined effects of receptor engagement and low $\mathrm{pH}$. These are complex concepts and some of the figure legends did not adequately explain what was represented. However, evolutionary constraints on viruses that affect entry, fusion inhibitors, new findings about steps preceding receptor engagement, and the basics of intracellular trafficking and uncoating were outlined clearly. This chapter focused on events at the cellular level, but also discussed virus entry in the whole-organism context. 


\section{Reverse transcription \& integration: Alan Engelman}

This well-written chapter provided a very solid introduction to current understanding of the enzymes and processes associated with early replication steps. The first figure provided a useful update to these topics and cellular context to these well-studied events. The coverage was comprehensive, clear and concise. Although somewhat more information was provided about integration than reverse transcription, this reflects the current research area of greater growth.

\section{Transcription, splicing \& transport of retroviral RNA: Tina Lenasi, Xavier Contreras \& Matija Peterlin}

This dense chapter described transcriptional- and splicing-level regulation of HIV-1 gene expression. The authors dived directly into the 'alphabet soup' of transcription factors without an overview: any reader who did not know at the outset that Tat is encoded by the virus, or what Tat does in general terms, would not learn that here. Another example of this chapter's confusing style was the use of the term 'PIC' (i.e., pre-initiation complex), which meant something very different here than it did in the preceding chapter or in the book's 'Abbreviations' section (i.e., preintegration complex). Although PIC was defined eventually, this was several pages after its first use and only in a figure legend. The HIV RNA processing portion of this chapter was noticeably better written that the rest. However, because the only virus described in this chapter was HIV-1, differences among retroviruses in properties, such as host RNA trafficking pathways employed, were not described here or anywhere else in the book. This chapter was the only place in the book where viral latency was discussed in any detail, and the treatment of this important topic was quite dated and limited in scope.

\section{Assembly \& release: Heinrich Gottlinger \& Winfried Weissenhorn}

This field has witnessed significant growth in the past decade or so. Key concepts in the structural biology of virus assembly were described clearly in this chapter. Lucid descriptions of how similar assembly processes are achieved by different types of retroviruses were also provided. 'Editorial comments' that let us know what new findings were groundbreaking (e.g., the discovery of tetherin as the host factor that inhibits viral particle release and is antagonized by $\mathrm{Vpu}$ ), what was unexpected based on previous findings and so forth, was helpful and contributed to making this chapter a pleasure to read. The addition of a figure or two to summarize and integrate complex concepts, such as the budding machinery involved in virus release, would have been useful.

\section{Transmission \& epidemiology: Hanz Lutz, Gerhard Hunsmann \& Jorg Schupbach}

This chapter provided a straightforward treatment of the topic, first presenting the host ranges of a large number of veterinary pathogens and these viruses' routes of transmission. This was followed by a useful overview of primate lentiviruses in the context of geography and host taxonomy, with a natural segue into the HIVs. The closing portions of this chapter introduced concepts of lentivirus zoonoses, HIV origins and geographic distribution of HIV strains. The chapter was rounded out by a description of the parameters that contribute to observed rates of HIV spread and to the prevention of its transmission. 


\section{Pathogenesis of oncoviral infections: Finn Skou Pedersen \& Annette Balle Sorensen}

Retroviruses were historically known as 'RNA tumor viruses', and this chapter described the three major mechanisms by which retroviruses cause cancer: by transducing host oncogenes, by serving as insertional mutagens, or through the tumorigenic activity of viral gene products, as exemplified by the transforming properties of Jaagsiekte sheep retrovirus's Env protein. This chapter provided a good balance of historical insight and molecular details into the pathogenesis of oncogenic $\alpha-, \beta$ - and $\gamma$-retroviruses. The chapter was scaffolded by clear and simple textbook-like descriptions, such as tables presenting examples of oncogenic retroviruses and viral oncogenes, without citations. These were interspersed with focused details on topics such as how transducing virus oncogenes function differently from their parental host alleles, and examples of the series of events involved in tumorigenesis for specific viruses or virus complexes. Brief treatment of more recent developments, such as high throughput analysis of common integration sites to identify new potential cancer genes, and the discovery of xenotropic murine leukemia virus-related virus as a candidate cancerassociated $\gamma$-retrovirus of humans, rounded out this very strong chapter.

\section{Pathogenesis of immunodeficiency virus infections: Guido Poli \& Volker Erfle}

This brief chapter seemed somewhat imbalanced. Approximately half of the text was very introductory and rather peripheral, with more space devoted to seemingly irrelevant information, such as how Th2 responses induce immunoglobulin class switching, than to topics that pertain to the pathogenesis of immunodeficiency viruses. Both of the principal content sections on immunopathogenesis and on neuro-pathogenesis of HIV and SIV were exceptionally brief and highly disorganized. Many assertions by the authors, such as that $\mathrm{p} 6$ interacts with tetherin, that extracellular Tat is important to pathogenesis (although this view was also espoused in chapter 16), and that, prior to AIDS, most scientists assumed human retrovirus infections would be easily eliminated, seem at best controversial or based on outdated information. This chapter was highly disappointing.

\section{Retroviral restriction factors: Jeremy Luban}

The chapter opened with a discussion of the mutagenic potential of retroviral integration the prevention of which Luban suggests is the reason host cells evolved the restriction factors that were the subject of this chapter, which target early, preintegration retroviral replication steps. The establishment of this premise was followed by a clear and concise introduction to Fv1, a mouse factor that restricts the replication of certain murine retroviruses, followed by an overview of APOBEC 3 factors, which were first discovered in humans as the factors targeted by HIV-1 Vif, and later shown to restrict the replication of a broad range of retroviruses and related elements. TRIM5 $\alpha$, which, similar to Fv1, is a capsid-targeting restriction factor, was then discussed in greater detail. Luban first presented the clues about this form of restriction that ultimately led to the identification of TRIM5 $\alpha$, which was revealed through genetic analyses and by patterns of host susceptibility and virus resistance. The author then moved on to describe the discovery of TRIM5 $\alpha$ as the factor that restricts HIV-1 replication in most simian cells, and to the remarkable story of how TRIMCyp fusions arose twice independently and became fixed in two distinct primate lineages. This was followed by a discussion of the restriction properties of TRIM5 $\alpha$ and, finally, by a summary of research exploring the structure, interactions and functional properties of TRIM5 $\alpha$, as well as how these properties might affect replication steps and mediate restriction. 


\section{Molecular vaccines \& correlates of protection: Stephen Norley \& Reinhard Kurth}

This chapter did a wonderful job of answering the question of why we still do not have a preventive AIDS vaccine, distilled to a level appropriate for a nonexpert. The authors walked through the myriad of vaccine approaches that have been conceived and attempted, starting with "classic" killed and live attenuated vaccines. The authors moved on to describe, one-by-one, the many different viral gene delivery systems and vaccination strategies that have been explored, the rationales for attempting each approach, any encouraging laboratory and/or clinical observations, and problems that have been encountered. The material was well organized and presented in a thought-provoking and highly readable fashion. The brief section on molecular vaccines to other retroviruses was packed with useful lessons learned from the challenges and successes achieved with vaccines for cat and horse viruses. The conclusion section provided a strong summary both of the chapter and of the challenges of developing an HIV vaccine.

\section{Y-retroviral \& lentiviral vectors for gene delivery: Michael Muhlebach, Silke Schule, Nina Gerlach, Matthias Schweizer, Christian Buchholz, Christine Hohenadi \& Klaus Cichutek}

Here, the authors started out by laying out the fundamental differences between $\gamma$-retrovirus and lentivirus vectors that influence their utility. The choice to include the prefix ' $\gamma$-' in this chapter's title was a welcome way to emphasize that lentiviruses and $\gamma$-retroviruses, as well as other retroviral genera, can all serve as the basis of retroviral vectors. This chapter was comprehensive, current and well written, but assumed a pre-existing knowledge of basics, such as what $\Psi$ and helper functions are and, thus, would have better served nonretrovirologist colleagues who might have wanted to use these vectors if it had opened with a paragraph or figure that described the basic cis- and trans-acting components. This chapter was packed with details of genetic engineering and other improvements that have been attempted, accomplished or are ongoing, which address retroviral vectors' safety, effectiveness and versatility. Therapeutic applications, and the successes and adverse outcomes of human clinical trials, were presented. Although this chapter's principal focus was on technology, the authors did a very good job of introducing sufficient background biology to provide the rationale for design features, including indicating where biological understanding remains weak.

\section{Nonprimate, mammalian \& fish retroviruses: Maribeth Eiden, Kathyrn Radke, Joel Rovnak \& Sandra Quackenbush}

This chapter reviewed the properties of retroviruses of cats, mice, koalas, fish and cattle, with a focus on the histories of their isolation, their host ranges/receptors, examples of crossspecies transmission, these viruses' phylogenetic relationships and the diseases they cause. The chapter was interesting and well written, but nonetheless seemed to be a bit of a 'boutique' chapter; somewhat unequal to most of the other chapters in scope and significance.

\section{Simian exogenous retroviruses: Jonathan Heeney \& Ernst Verschoor}

Having read this book straight through from the beginning, it seemed a bit repetitious to once again be told about the seven genera of retroviruses, the morphology of particles, genetic organization and replication, and history starting with Peyton Rous, and Ellerman and Bang. Much of what was presented in this chapter mirrored the chapter 8 content, and 
the two could have been combined. However, this chapter was the principal source of information on foamy viruses, the only non-Orthoretroviridae retrovirus genera. The conclusions section focused on retroviral restriction factors, which were not mentioned in the chapter other than in this section, and stated that these are interesting times. Indeed!

\section{HTLV \& HIV: Marvin Reitz \& Robert Gallo}

This chapter opened with the discovery of human T-cell leukemia virus (HTLV), describing how advances in cell culture and retrovirus detection converged to allow the discovery of HTLV-1 in the late 1970s, and how epidemiology strengthened the putative causative association of HTLV-1 with a subset of leukemias in southern Japan. This introduction was followed by an overview of HTLV virology and pathogenesis. Next, a section on HIV-1 introduced the discovery of this virus, followed by a concise description of HIV virology, replication, host interactions and pathogenesis.

\section{Conclusion}

Although not explicitly stated, based on its choice of contents and promised scope, Retroviruses: Molecular Biology, Genomics and Pathogenesis aimed to replace the venerable but outdated 'bible of retrovirology', Cold Spring Harbor Press' 1997 Retroviruses monograph. Although there are some very good chapters, this lofty goal was not met. The book appeared to be far less well edited than most good scientific journals, in that chapters were highly uneven and some were rife with misstatements (e.g., calling exogenous viruses endogenous viruses), poor writing and a high density of grammatical errors, while other chapters were very strong. The index was brief and did not capture as much of the contents as would have been useful. In general, the better the chapter, the less current it appeared to be, suggesting some chapters were written early and well (an impression confirmed by one author who stated that October 2007 was the present time), while others appeared to have been tacked on later by authors less invested in the project.

Some chapters only discussed HIV-1, while others provided a more integrated view of retrovirology. Koala and cat retroviruses were covered in chapter after chapter, but avian viruses were barely mentioned. Although the 'Preface' apologizes in advance for the unavoidability of some repetition, a better job of coordinating which author was responsible for what information could have reduced the extent to which many areas were covered shallowly again and again, while others were absent or severely outdated. For the most part, this left the impression that the content of the chapters was based more on what the authors could easily deliver than on what the reader needed to know. The lack of good information about HIV pathogenesis was particularly unfortunate.

In this era of metastasizing review journals, what needs justify a book? The editors asserted that this book would serve both to bring newcomers up to speed and to provide a singlesource reference for established retrovirologists. This volume does contain several good source chapters, but it is not until the final chapter that we are provided something resembling the introductory information that one would convey about retroviruses in one or two lectures to medical students (e.g., an overview of retroviral replication cycles). Furthermore, even in the final chapter, no simple figure of replication from entry to release was provided. Other topics from introductory lectures, such as HIV-1 dynamics and patterns of CD4 cell decline over the course of an HIV-1 infection, were nowhere to be found. Thus, although many of the chapters were well written and informative, and a couple achieved the goal of taking the reader from the subfield's basics through the current state of knowledge, this book did not include an introductory chapter that would serve as a starting point for the rank beginner. 
In summary, this is an uneven collection of chapters. Chapters on certain replication processes, and on oncoretroviruses, veterinary pathogens, vaccines and vectors, were very good, while some of the other chapters were significantly less strong. Many chapters will be useful to a junior investigator with a pre-existing strong understanding of retroviruses in overview. Several of the areas of most rapid growth in the field are covered well through to 2007 , with limited updated information and, thus, provide a snapshot of this critically important and fast-moving field.

\section{Acknowledgments}

This work was supported by the NIH (R21AI080276). 